\title{
El Negrojudio en país madre de Ellen Ombre. Retrospectivas al siglo XVIII en el Caribe
}

\author{
The Negrojudio en País Madre by Ellen Ombre. Retrospective \\ to the XVIII Century in the Caribbean \\ O Negrojudio en país madre (Pretojudeu em país mãe) de \\ Ellen Ombre. Retrospectivas ao século XVIII no Caribe
}

\section{Ineke Phaf-Rheinberger}

HUMBOLDT-UNIVERSITÄT ZU BERLIN, INSTITUT FÜR ASIEN - UND AFRIKAWISSENSCHAFTEN (IAAW), ALEMANIA

Investigadora asociada de la Humboldt-Universität, Alemania. Doctora

Lateinamerika-Institut, Freie Universität Berlin. Es autora de Zur

Architektur von Ricardo Porro in Vaduz und Havanna (Coleba, 2004),

La Belle Caraïbe. The Art of José María Capricorne (Wissenschaftlicher

Verlag Berlin, 2005), The Air of Liberty. Narratives of the South Atlantic

Past (Rodopi, 2008) y editora de Historias enredadas. Asimetrías con

vista al Atlántico (edition tranvía, 2011); con Tiago de Oliveira Pinto,

AfricAmericas. Dialogues, and Sounds (Vervuert - Iberoamericana, 2008);

con Michael Mann, Beyond the Line. Cultural Narratives of the Southern

Oceans (Neofelis 2014). Correo electrónico: phafrhei@cms.hu-berlin.de

Artículo de reflexión

Primera publicación en: El Caribe y sus diásporas: Cartografía de saberes y prácticas culturales, Anja Bandau y Marta Zapata Galindo (Eds.). Madrid: Verbum, 2011. 166-184.

Documento accesible en línea desde la siguiente dirección: http://revistas.javeriana.edu.co

doi: 10.11144/Javeriana.cl19-38.enpm 


\section{Resumen}

En la novela Negrojudio en país madre de Ellen Ombre se trata de memorizar toda la trayectoria de la descendencia familiar a partir del presente hasta finales del siglo XVIII del carácter principal femenino. Las retrospectivas se realizan a lo largo de la descripción de un día en su vida en Amsterdam en 2000, cuando ella se separa de su marido. La coincidencia de las orientaciones religiosas judías y cristianas se van alternando de acuerdo con la progresión de la narración demostrando sus asociaciones diferentes de status social en una sociedad con pasado esclavista.

Palabras clave: drama multicultural contemporáneo; neologismo negrojudío; migración Surinam-Europa; orientación religiosa; identificación con el papel de la mujer

\section{Abstract}

In the novel Negrojudio en país madre by Ellen Ombre, she tries to recall the whole path of the ancestry, from the present time to the late XVIII century, of her main female character. The retrospectives are made during her description of a day of her life in Amsterdamn, in the year 2000, after her separation from her husband. The coincidence of the Jew and Chrsitian religious orientations are alternative choices, as the narration progresses, showing their different associations with social status in a society with a past related to slavery.

Keywords: multicultural contemporary drama; negrojudío neologism; migration SurinameEurope; religious orientation; identification with the female role

\section{Resumo}

No romance $\mathcal{N}$ egrojudio en país madre de Ellen Ombre trata-se de memorizar a trajetória toda da descendência familiar a partir do presente até finais do século XVIII do carácter principal feminino. As retrospectivas são realizadas ao longo da descrição de um dia na sua vida em Amsterdã no ano 2000, quando ela se separou do marido. A coincidência das orientações religiosas judaicas e cristãs vão se alternando de acordo com a progressão da narração demostrando as associações diferentes de status social numa sociedade com passado escravista.

Palavras-chave: drama multicultural contemporâneo; neologismo pretojudeu; migração Suriname-Europa; orientação religiosa; identificação com o papel da mulher

RECIBIDO: 11 DE ABRIL DE 2014. ACEPTADO: 1 DE JUNIO DE 2014. DISPONIBLE EN LÍNEA: 01 DE JULIO DE 2015

\section{Cómo citar este artículo:}

Phaf-Rheinberger, Ineke. "El Negrojudio en país madre de Ellen Ombre. Retrospectivas al siglo XVIII en el Caribe". Cuadernos de Literatura 19.38 (2015): 202-220. http://dx.doi.org/10.11144/Javeriana.cl19-38.enpm 
Al comienzo Del nuevo milenio, los Países Bajos se despertaron de repente de su ilusión de ser un país con una tradición extraordinariamente tolerante. Este despertarse se inauguró con la polémica alrededor de un artículo del periodista Paul Scheffer sobre el drama multicultural ocasionado por el retraso dramático de los hijos de los migrantes en el sistema educativo. Esta publicación en el diario $\mathcal{N} R$, del 29 de enero de 2000 , fue motivo de una verdadera avalancha de reacciones dando muestra de un sentimiento general de inseguridad. Dos años después, el 6 de mayo de 2002, ocurrió el asesinato del político Pim Fortuyn y el 2 de noviembre de 2004 de Theo van Gogh, el cineasta.

En medio de este clima de tensiones entre los alóctonos y los autóctonos ${ }^{1}$ se sitúa la trama de $\mathcal{N}$ egrojudío en país madre (Negerjood in moederland, 2004), la primera novela de Ellen Ombre. Su protagonista Hannah Dankerlui es una 'negrojudía', migrada de Paramaribo a su "país madre" en la década de 1960, cuando Suriname era todavía una colonia holandesa (hasta 1975). Ombre narra un día en el invierno de 2000, el momento que Hannah deja su casa en el grachtengordel (cintura de canales) en el centro de Amsterdam buscando refugio en el Bijlmermeer, un suburbio al sudeste de esta ciudad.

La autora encuadra los polos de los conflictos novelescos en un marco específico. Por un lado, se encuentra la mezuzá, la palabra hebrea de un receptáculo adherido a la jamba derecha de los pórticos de las casas judías que alberga un pergamino con algunos versículos de la Torá. Y por otro hallamos el tapu, una palabra sranan indicando un amuleto que relaciona con la religión winti de los afrosurinamenses ${ }^{2}$. Ambos objetos, derivados de religiones tan diferentes, ofrecen protección contra las malas influencias. Sin embargo, cuando Hannah piensa en el hábitat de su familia, lo percibe como "el apartamento deprimente: la mezuzá, como un tapu en el poste de la puerta" (Ombre, Negrojudío 8) ${ }^{3}$. La analogía entre estos dos símbolos de protección programa los problemas internos. La madre Emma Henco desciende de una adinerada familia sefardí en Paramaribo, mientras que el padre August Dankerlui es hijo de descendientes de esclavos. Ambos fueron miembros de la iglesia de los Hermanos Moravos, altamente reconocidos y respetados por su trabajo educativo entre los afro-descendientes y los indígenas a partir del siglo XVIII en Suriname.

1 Estas categorías se aplican en Holanda para distinguir entre los alóctonos (alochtonen), los hijos de los migrantes, mientras que los autóctonos (autochtonen) representan a los hijos de una familia holandesa.

2 Sranan es la lengua criolla (creole language) y winti la religión afrosurinamense: ambas arraigadas en Suriname desde los inicios del siglo XVIII.

3 de beklemmende flat: de mezouza, als een tapu aan de deurpost (Ombre 2004, 8). 
La novela de Ombre contiene rasgos autobiográficos. Al igual que su protagonista, nació en Suriname, llegando como muchacha a Holanda a principios de los años sesenta. Además vivió durante décadas en una casa-monumento parecida a la Herengracht, uno de los tres canales más antiguos en el centro de Amsterdam, a el grachtengordel, con su exmarido, el profesor de sociología, Abram de Swaan. La palabra negerjood del título, sin embargo, no se encuentra en el Diccionario de la Real Academia Holandesa (Diccionario Van Dalen) aunque cada holandés entiende su sentido. Al colocar esta palabra en un lugar tan destacado como el título de su novela, Ombre le otorga una importancia extraordinaria en su aspiración de localizar las asociaciones negativas alrededor de su presencia, al igual que hizo Aimé Césaire al crear la négritude. En un libro sobre temas etnográficos, literarios y del arte en el siglo pasado, James Clifford observa que mediante esta estrategia de neologismos, Césaire construye sus textos "from a debris of historical and future possibilities" (175), como "a concrete, not abstract, coming to consciousness" en el Caribe que se manifiesta como un "language process" (177). Clifford señala que Césaire acuña este neologismo a fin de excavar una historia tabuizada y poco explícita. De acuerdo con esta intención, he tratado de conservar el sentido del neologismo negrojudío en la traducción al español, pese a que las traducciones al francés (2006) y al inglés (2007) han optado por otra solución. Al darse cuenta de esta estrategia de neologismos, se explica la depresión que Hannah experimenta al sentirse confrontada con la mezuzá y el tapu en su historia familiar, el leitmotiv de la trama novelesca.

Conforme al dilema entre la mezuzá y el tapu en la familia, Ombre retrata una relación compleja entre hija y madre creando así un efecto de déjà-vu, de familiaridad en los lectores que lo identifican inmediatamente como una situación observada con frecuencia en su propio medio ambiente. Su desenvolvimiento se desarrolla sobre el fondo de los debates polémicos en Holanda durante el invierno de 2000 referiéndose al drama multicultural: por un lado, los alóctonos pertenecen al grupo de migrantes "de color" de origen maroquí, turco, antillano o surinamense, mientras que los autóctonos son los holandeses blancos.

\section{La historia de la diáspora judía}

Hannah Dankerlui nació en 1952 en Paramaribo. En el invierno de 2000, pone fin a los 31 años de su matrimonio en Amsterdam. Mientras que el hilo narrador sigue las etapas de su ruta desde la mañana hasta la noche, se alternan recuerdos de otros tiempos en retrospectiva, como hilos sueltos de memorización. De esta manera, se explican los detalles de los problemas afectivos de la protagonista. 
En primer lugar, se trata de las razones del fin del matrimonio de Hannah con Chaim, un profesor holandés. Para ella, ser una negrojudía de Suriname desempeña un papel importante. En la casa de los Dankerlui-Henco existe un tabú en cuanto a este tema. La madre judía y "casi blanca" no quiere hablar de su pasado mientras que el padre resulta ser más abierto. Ambos descienden de los dos grupos migrantes más antiguos que llegaron a la colonia-plantación holandesa en América.

El objetivo principal de la colonización fue administrar una economíaplantación, lo que implicaba ser involucrado en la Trata con África. Günther Böhm menciona en Los sefardíes en los dominios holandeses de América del Sur 1630-1750 (1989) los privilegios concedidos por los holandeses a la nación portuguesa, como solían caracterizarse los sefardíes a sí mismos. Les ofrecieron a sus miembros la más amplia libertad religiosa, el derecho de construir sinagogas y escuelas, el derecho de comprar terrenos así como de tener sus cementerios propios si migraban a la nueva colonia en la Costa Silvestre de América del Sur: A aquéllos que habían regresado en 1654 desde Recife, Pernambuco, a Holanda, se les garantizaba, además no ser procesados por deudas adquiridas en Brasil, durante su permanencia en este país, con las tropas holandesas. Para hacer más atractivos aún estos privilegios, se ofreció a los judíos transporte liberado, para ellos, su familia y sus pertenencias; materiales para edificar sus casas, cultivos para la tierra y autorización de portar armas para su defensa personal. (110)

No obstante estos privilegios, mientras tanto, no se dejaron esperar los conflictos con el gobierno de la colonia. Ana Crespo (2006) escribe en América desde otra frontera. La Guayana Holandesa (Surinam): 1680-1795:

Algunos testimonios de primera mano sobre la convivencia de los distintos grupos socio-étnicos en el seno de la sociedad de Surinam apuntan a la existencia de claros conflictos entre los colonos judíos y los protestantes neerlandeses. Los primeros vivían en la denominada "Sabana de los judíos", construyendo sus plantaciones aisladamente del resto. Constituían la Joodsche Natie y sus empeños en mantenerse aislados del resto de los colonos fue motivo de problemas para las instituciones coloniales. (121)

Estos problemas culminaron en el siglo XVIII, período de gran prosperidad en que el país resultaba ser cada vez más el escenario de una permanente guerra interna contra los cimarrones, los desertores de la colonia-plantación. Durante la crisis económica en la década de 1770, la nación portuguesa perdió 
su autonomía en la Sabana Judía. Muchos de sus miembros tenían que migrar a Paramaribo o al exterior.

Obviamente los privilegios de los migrantes sefardíes eran relativos. En contraste, los de los esclavos de origen africano eran nulos. Entre ellos, a partir de 1735, los Hermanos de la Iglesia Morava habían iniciado su misión. A diferencia de las otras religiones protestantes, comenzaron a predicar entre los esclavos en Paramaribo así como entre los amerindios y los cimarrones del interior. No solo les enseñaban el Evangelio sino además lo hacían hablando en sus lenguas. Predicar en sranan en Paramaribo en el siglo XVIII fue una cosa extraordinaria que llamaba mucho la atención. Pese a que todos lo hablaban de un modo u otro, era una lengua de poco prestigio por ser hablada por gente de ascendencia africana y esclavizada. Los Moravos fundaron además escuelas en momentos en que el sistema de educación fue prácticamente inexistente. Así no es de sorprender que los primeros poetas en sranan -Papa Koenders y Trefossa-fueran miembros de la Iglesia Morava y se atribuye a ella el mérito de haber contribuido decisivamente al reconocimiento del sranan como una lengua apropiada para producir literatura. Según los lingüístas, el sranan se desarrolló en el siglo XVIII como la lengua más importante para la comunicación entre los diversos grupos de la población y muchas fuentes consultadas derivan de los archivos moravos (Berg 2008).

Ombre emprende en su novela un viaje de exploración, de una búsqueda de las raíces del sentido de las connotaciones negativas referentes a la presencia del negrojudío. Hannah, en Holanda a partir de los doce años, cuando es alumna de la escuela secundaria, empieza a ganarse algún dinero los sábados en una casa de ancianos judíos, sobrevivientes del Holocausto, en Amsterdam. El Sabbath no le ofrece ningún impedimento para trabajar porque ella es una no-creyente, una shabbesgoj. Una anciana le regala una menorá, un candelabro de plata de siete brazos, uno de los símbolos más tradicionales del judaísmo. En casa, Hannah regala la menorá a su madre viendo la alegría con que esta se acuerda de objetos similares en su casa familiar en Paramaribo. De este modo, la menorá constituye un primer lazo positivo con su vida anterior de la que nunca habla, como si fuera un episodio tabuizado. El entusiasmo de Hannah motiva al padre a empezar a coleccionar datos sobre la familia de su mujer mientras que la menorá consigue ocupar un lugar céntrico en la sala del apartamento.

$\mathrm{Su}$ trabajo como shabbesgoj le estimula a Hannah a aprender el hebreo y devenir miembro de la asociación zionista Haporzim con la perspectiva de ir a trabajar en un kibuz en Israel. Se enamora de Gideon que sale a Israel poco antes de la guerra de seis días en 1967. Simultáneamente sin embargo, Hannah se siente atraída hacia los pleiners, los jóvenes no-conformistas vestidos de negro y amantes 
del jazz y blues. Suelen reunirse en los cafés alrededor del Leidseplein. Cuando se decepciona con la organización rígida del Haporzim, Hannah encuentra aquí en el famoso café Americain a otro hombre judío, Chaim. Entiende esta relación y el siguiente matrimonio gracias a lo que su madre le había instruido, es decir a acomodarse a una situación en la que ella se comporta dócil (gedwee 67), dedicándose por completo al bienestar de su marido.

Chaim es antropólogo y trabaja en la universidad de Amsterdam. Le gusta viajar con frecuencia para no faltar a los congresos importantes de su disciplina. Toma todo tipo de libertades con otras mujeres "de color" sin importar su nacionalidad. La madre de Chaim pertenece al circuito intelectual activista que siempre interviene a favor de los derechos de la mujer y de otras iniciativas progresistas. En este circuito se acostumbra conversar sobre temas como política, enfermedades genéricas, orgasmos y el bien o el mal en la Segunda Guerra Mundial. No existe ningún tabú de acuerdo con el lema: "todo debe ser posible" (alles moet kunnen). Su matrimonio y su subsiguiente estudio universitario le introducen a Hannah en este medio ambiente tan "abierto", del cual se siente radicalmente apartada cuando este le otorga la estampa de ser alóctona.

\section{El cuaderno del padre}

La separación de Chaim por lo tanto implica la separación del mundo de los autóctonos. Ombre organiza su libro en relación a esta ruptura. Hannah sale de la casa-monumento en el centro con cuatro maletas y dos bolsos por la mañana para ir buscándose albergue en el apartamento de una surinamense que vive en el sureste de Amsterdam. La autora describe la topología de esta ruta con gran precisión. Desde el Herengracht, Hannah camina hacia la torre De Munt -un edificio construido en el siglo XVII para acuñar monedas- donde toma un taxi a la entrada del metro Waterlooplein. Aquí toma el metro a la estación Kraaiennest, cuya salida queda cercana a su amiga con la que se queda por la noche.

Los 16 capítulos narrados en tercera persona contienen fragmentos sobre experiencias cruciales en la niñez en Paramaribo, los primeros años en Holanda, la vida íntima con Chaim, las visitas a su familia o sus conversaciones terapeúticas con psicólogos. Hannah se ve confrontada con el trauma de la Segunda Guerra Mundial, $\tan$ fresco en la memoria holandesa de los años sesenta. Sus ideas sobre la liberación sexual contrastan significativamente con las ideas de su madre que aspira mantener el control absoluto sobre ella. Hannah encuentra su "felicidad" -según la madre- al casarse con un hombre holandés bien situado y sin pretensiones. A partir de ese momento, Emma ha cumplido su deber maternal y se muestra indiferente en cuanto a la vida matrimonial de su hija. 
El contacto con el padre es diferente. Tiene intereses parecidos. Como bibliotecario sabe estimar el valor de un texto. Sigue la política holandesa con atención, comunicando a Hannah su interpretación desde una perspectiva surinamense. Para obtener información sobre la familia de su mujer establece una correspondencia con un archivista en Paramaribo. Incluye recortes, cartas, anotaciones, extractos de documentos oficiales, fotos y artículos en un cuaderno en cuya portada escribe "Para Hannah".

Al leer este cuaderno después de su muerte, Hannah se entera de que su depresión acerca de la analogía entre mezuzá y tapu se deduce del problema de los sefardíes con los esclavos y "libres" en Paramaribo en el siglo XVIII. Habiendo sido educados en la religión hebrea, no les fue concedido un status de igualdad en la comunidad hebrea. Por consiguiente, fundaron una sinagoga propia e hicieron una petición al gobernador de la colonia para que interviniera poniendo fin a esta injusticia. El conflicto escalaba cuando uno de sus miembros más destacados murió y les fue prohibido enterrarle en el cementerio entre los otros miembros -sin ascendencia esclava- de la nación portuguesa en Paramaribo.

El padre ha incluido asimismo los detalles de la historia de los antepasados de Emma Henco en Suriname. Comenzó con Isaac y Lea Cohen al establecerse en la Savannah Judía a finales del siglo XVIII. Habían migrado de Francia a Amsterdam donde recibían el apoyo de la nación portuguesa para migrar a Suriname bajo la condición de no volver por lo menos durante quince años. En aquellos tiempos, la Sabana Judía ya estaba casi abandonada y no se parecía más a la conglomeración de ricos proprietarios de antaño.

El matrimonio Cohen vive bajo un signo trágico. Uno de sus hijos, Samuel, nacido en 1812, tiene una relación sexual con la misma esclava favorita de su padre. Nueve meses después nace una bebé "casi blanca", que al cumplir dos años será regalada a una familia en Paramaribo a fin de liquidar deudas. La madre esclava se suicida por dolor. Un mes después de deshacerse de esta pequeña esclava, la madre de Samuel -Lea Cohen- da a luz a una hija, Louise, ¡una judía mulata!, un verdadero escándalo. La herencia hebrea es matrilinear. Ombre sugiere a través de la organización de su texto -sin decirlo explícitamente- que el padre pudiera haber sido el esclavo Manus que trabajaba como esclavo doméstico en la casa. Lea muere en seguida después de haber dado a luz y muy avergonzados, Isaac y Samuel se mudan con sus esclavos a Paramaribo. Aquí venden a la bebé y a Manus a una negra libre (vrije negerin). Años después, al comenzar Louise una relación con un negro libre de padre judío, esto le ocasiona el odio de su patrona ya que esta hubiera querido tener amoríos con ese hombre. Su venganza es atroz. Pide un permiso oficial a la Corte para castigar a su esclava en público, un juicio 
cuya realización se comenta entre los habitantes de Paramaribo durante mucho tiempo debido a la demostración de una crueldad extraordinaria.

El padre ha incluido un árbol genealógico en el cuaderno con la anotación de que "el lazo de sangre se trasmite a través de la madre" (Ombre, Negrojudío $169)^{4}$. Evidencia que esta Louise es la tatarabuela de Emma Henco. Por lo tanto, la lectura del cuaderno da a Hannah la certeza de que ella, como Chaim, es judía. Tiene el efecto de una revelación ya que desde el principio, Ombre enfatiza las diferencias entre estos dos personajes de su novela. Cuando la primera visita de Chaim a la casa Dankerlui-Henco, este se comporta como un verdadero bakra (blanco) que no tiene ninguna dificultad de conversar sobre cualquier tema y todo sin hacer pausas. A la madre le encanta su informalidad, mientras que el padre queda más reservado. Hannah observa que ambos dan muestras de un comportamiento artificial, por miedo de aparecer como inferiores, una herencia del pasado que sobre todo se expresa en su manera de hablar holandés:

[...] esconderse tras una lengua artificial. Camuflaje debido a una inseguridad profundamente arraigada. Quien hablaba mal el holandés era considerado como atrasado, estúpido. Por aquel entonces los esclavos negros tenían la fama de ser infantiles, porque tenían que hablar con sus dueños en lenguas que no conocían. Y la mayoría de aquellos tenía el interés de mantener este abismo lingüístico. Lengua era clase. Imitación una estrategia de sobrevivencia de los negros. (Ombre, Negrojudío 69)

La manera de hablar o, según Clifford el "language process", es un punto neurálgico. Este modo de hablar sin pausas y con tono agitado le distingue a Chaim del habla de los surinamenses. Ombre describe con esmero el talento con que la abuela de Hannah le cuenta fábulas y cosas del pasado en su estilo reposado y reflexivo de conversación, al igual que el padre al comunicarle a ella sus opiniones políticas. Su lengua nativa es holandés, lo que explica que cuando le preguntan a Hannah por su identidad alóctona, como primera generación migrante, ella se siente extremadamente ofendida. No obstante que habla holandés como ellos, los autóctonos le dejan enfilarse en el otro campo. Este efecto aumenta aún más en una última conversación con Chaim, cuando Hannah le revela su

$5 \quad[.$.$] zich verschansen achter verheven taal. Camouflage vanuit diepgewortelde onzekerheid.$ Wie gebroken Nederlands sprak werd achterlijk, dom gevonden. Negerslaven hadden destijds de faam kinderlijk te zijn, omdat ze moesten spreken met hun meesters in talen die ze niet beheersten. En die meesters hadden er alle belang bij de taalkloof in stand te houden. Taal was klasse. Mimicry een overlevingsstrategie van de negers (Ombre, Negrojudío 69). 
identidad judía. Tiene un resultado dramático. Al escucharlo, Chaim le confiesa que si lo hubiera sabido nunca se hubiese atrevido a tratarle de la misma manera por ser incapaz de ofender a una mujer de su propia condición.

El dejar atrás a la grachtengordel significa abandonar el barrio construido en el momento de mayor expansión sobre el globo en el siglo XVII para ir al Bijlmermeer, un suburbio construido a finales de los años sesenta. El Bijlmermeer se llama blaka foto (ciudad negra) en sranan y es el destino ideal en el imaginario de muchos surinamenses. Les posibilita convivir con una confluencia de migrantes africanos, lo que produce momentos de gran emoción. Los ghaneses por ejemplo, entienden la lengua litúrgica del winti, en cuyas ceremonias se establece un contacto con los ancestros. Aparte de esto, en este suburbio Hannah se entera de un detalle clave del matrimonio de sus padres. La amiga le repite la frase con la que la madre de August Dankerlui, la abuela de Hannah, solía expresar su opinión con respecto a la relación de su hijo con Emma Henco: "Un judío blanco la embarazó antes de abandonarla y, luego, el hombre negro tapó su vergüenza [por casarse con ella]" (Ombre, Negrojudío 186, 189) ${ }^{6}$. Al final de la novela se entiende el origen del comportamiento neurótico de la madre con su hija. Ella misma era una niña adoptada y abusada en su familia y en un momento difícil, August Dankerlui la "salvó" del desprecio social por contraer matrimonio con ella.

\section{¿Con qué relacionarlo?}

Con esta frase clave en sranan, como expresión de un momento de máxima intimidad, Ombre se incorpora en una reciente tradición narrativa de alternar el holandés con el sranan como estrategia para interpretar el pasado y presente de Suriname (Phaf-Rheinberger 2001). Valga la pena llamar la atención sobre este medio estilístico, ya que el sranan nunca fue la lengua oficial del país. Por otro lado, también se detecta una influencia de la literatura holandesa contemporánea, ya que el contenido de Negrojudío en país madre inmediatamente recuerda la novela-bestseller de Connie Palmen, IM. Ischa Meijer (1998). El yo-lírico de Palmen retrata su historia de amor con otro intelectual, igualmente promiscuo y judío, el periodista holandés, Israel Chaim (Ischa) Meijer (1943-1995).

En contraste con Palmen, el estilo de Ombre es muy poco emocional. Escribe con un tono de understatement al compartir sus temas de viaje, migración y movilidad transatlántica con otro autor, Caryl Phillips, un inglés nacido en el Caribe que aplica este método de retrospección y contrastes para curar las heridas del pasado (Gyssels 2003; 2004). Ya antes de haber publicado su primera novela,

6 A wit' Dyu bin libi eng nanga here dan wan blakaman kon tapoe eng syen. 
Ombre era una escritora respetada con tres libros de cuentos y un reportaje de viaje a África, ahora en su segunda edición ampliada. Los periodistas suelen caracterizarla con adjetivos como suspicaz, cautelosa, recelosa, desconfiada y cuidadosa, como una persona que controla sus emociones en público, enemiga de generalizaciones o categorías. Al retratar a Ombre, Silvio Torres-Saillant la describe como:

$[\ldots]$ a short fiction and autobiographical travel writer, [who] has explored the sociocultural contrast between Caribbeans and Europeans, while also reflecting on the contemporary remnants of the triangular trade that once linked Holland, Africa, and the Caribbean. (Torres-Saillant 196)

Ombre tiene una interpretación muy particular de este lazo triangular y comercial del pasado, ya que el tema que le apasiona es el de la "ayuda financiera para el desarrollo" a los países "subdesarrollados" o del "Tercer Mundo". En uno de sus cuentos más tempranos, "El precio de la ayuda" (De prijs van de hulp), Ombre representa una escena en una discoteca en Tchaourou, una pequeña ciudad en Benin. Visita un bar en compañía de un representante holandés de esta "ayuda". El barman la saluda a ella como a una "Camarade Cubain, Brésilien", debido a su piel negra pese a, obviamente, no ser africana. En Benin se desconoce la existencia de la "mobilidad colonial" entre Suriname y Holanda pero gracias a Fidel Castro, Cuba sí existe y el Brasil también gracias al Carnaval. Ambos países han invertido en Benin, como también Holanda, pero Holanda -para el barmanes un país blanco. Como gesto de amistad con ella, el barman pone un bolero de Celia Cruz en el tocadiscos, sin saber que fue una de sus cantantes favoritas cuando era joven en Paramaribo. Ella no baila sino le muestra su familiaridad con esta música. Mientras tanto, el holandés baila con un beninés de tal manera que da prueba de su incomprensión, su desprecio y su indiferencia total ante la población local.

En Negrojudío en país madre, Hannah Dankerlui realiza una ruptura definitiva con esta mentalidad "autóctona" reforzándola con el efecto de un orgasmo feliz al final. La liberación corporal le permite deshacerse por completo del corset en que había tenido que vivir. En este último momento de gran emoción con que la novela termina, se plantea la posibilidad de otro futuro alternativo, para cuya realización blaka foto desempeña un papel prioritario. Simboliza que Hannah, que siempre se había entendido a sí misma como una holandesa de Suriname, como resultado del drama multicultural opta por insertarse en el otro campo, el mundo de los alóctonos. 
Negrojudío en país madre de Ombre ilustra la perpetuación de un dilema heredado de la colonia holandesa (Phaf-Rheinberger 49-90). No obstante, traspasa las fronteras de Suriname y Holanda extendiéndose hacia una geografía mucho más amplia. Se plantea este tema delicado con mucha cautela en varios estudios recientes, cuyas observaciones concuerdan con las estrategias de exploración y precisión narrativas aplicadas por Ombre. Nicholas Mirzoeff reune una serie de ensayos sobre las representaciones visuales de africanos y judíos en Diaspora and Visual Culture. Representing Africans and Jews. Representing Africans and fews (2000). En la introducción Mirzoeff aboga por una concepción de identidades múltiples que, cuando son visualizadas, logran producir el efecto de "placer". En su opinión los momentos de optimismo, en confrontación con los momentos dramáticos y violentos, así como los episodios contradictorios, polifónicos, y utópicos marcan el camino de la representación visual de la diáspora. A su vez, Emily Miller Budick analiza en Blacks and Jews in Literary Conversation (1998) una serie de textos -cartas, ensayos, y literatura- de autores como Toni Morrison, Ralph Ellison, James Baldwin o Hannah Arendt. Budick se pregunta por sus diversas interpretaciones del Holocausto y de la esclavitud en América. Para entender más la historicidad del dilema del negrojudío, sin embargo, la obra de Jonathan Schorsch, Fews and Black in the Early Modern World (2004), es fundamental. Este teólogo analiza los criterios aplicados a la esclavitud en las comunidades judías desde el siglo XVI hasta el XVIII. Arguye que

[...] only Curaçao and Suriname, both with larger numbers of Jews holding slaves and engaging in plantation agriculture, produced some such legislation, traces of which remain extant. (218-219)

Schorsch no reproduce las legislaciones en cuestión sino menciona la presencia de la mezuzá en las casas de "gente de color" en Paramaribo en los años 1940s:

Many colored people of Paramaribo [...] held the mezuza fixed by Jews on their doorposts in "high esteem" as a kind of protective charm. Mezuzot could thus be found affixed in the correct place on the homes of non-Whites, but empty of the parchment with biblical passages required by Jewish law. Often "some text, in Dutch, from the Old or New Testament, is placed inside. Even non-holy texts are used. Such a text must always have some impressive wording, or a well-known proverb". These syncretistic uses of the mezuza and other ritual objects or rites taken from Jews show, however, that we have left the realm of a believing practice of Judaism for even more nebulous regions of affinity, empathy, and mimicry. (229-230) 
Es interesante que esta observación de Schorsch coincide con el esfuerzo por parte de artistas, escritores y críticos de echar más luz sobre estas "zonas nebulosas", de "afinidad, empatía e imitación". En el caso de Ombre, la luz esclarecedora ilumina el banlieu de Amsterdam que, en el año 2000, se ha convertido en el destino de los migrantes de países que fueron colonizados. Connotan este barrio con un circuito de circulación del saber, de un intercambio informal y del reconocimiento de detalles compartidos en sus culturas a través de la historia a ambos lados del océano. Relaciona con el winti y el tapu e incluso el nombre del Bijlmermeer en sranan - blaka foto - es instrumental al desmitificar la longue durée de un sentido de inferioridad que se remonta al pasado colonial.

La novela de Ombre no es la única que explora el siglo XVIII. Su paisana Cynthia McLeod, realizó un estudio extensivo sobre la vida de Elisabeth Samson (1996), una negra libre en Paramaribo, propietaria de plantaciones y de esclavos que vivió de 1715 a 1771. Elisabeth viajó varias veces al país madre para hacerse cargo de sus negocios además de mantener una extensa correspondencia con sus agentes en Holanda. Es protagonista en la ruta Amsterdam-Paramaribo entre 1740 $y$ los 1780 s, caracterizada por Jonathan Israel como "one of the most important in eighteenth-century Dutch long-distance navigation" (945). Al reconstruir la vida de Elisabeth de manera novelesca, McLeod arguye que Elisabeth, no obstante ser rica, nunca logró ganarse el respeto en la sociedad urbana de su tiempo debido a lo que los brasileños llamaron "um defeito do cor" (Gonçalves 2006) en términos oficiales, es decir el defecto de color, de ser negra. No existe ningún retrato de ella así que no se sabe nada de su apariencia, solo que pasaba su vida en una casa grande rodeada de objetos lujosos cercana al Waterkant, el barrio portuario de Paramaribo. Pese a su éxito -siendo negra logra casarse oficialmente con un europeo blanco- Elisabeth muere decepcionada porque este casamiento solo se proclama a nivel público pero no le da satisfacción a ella en su vida privada.

La trama de la novela La negra libre Elisabeth, prisionera de color (De vrije negerin Elisabeth. Gevangene van kleur, 2000) se sitúa en el marco de la "movilidad colonial" de los "libres" -de ascendencia esclava y origen africano habiendo nacido en libertad o manumisionados- en Paramaribo, sujeta a las restricciones normativas de la administración. McLeod, al igual que Ombre, enfatiza el carácter migratorio de la Guayana holandesa en el siglo XVIII. Elisabeth y sus familiares son nativos que -con los judíos- pertenecen a los grupos residentes más antiguos. Sus partners, mientras tanto, con quienes viven en concubinato o en matrimonio legal, son migrantes de Europa. 


\section{El siglo XVIII en la novela del Caribe}

El momento supremo del combate radical contra este tipo de colonia-plantación se manifiesta en la Revolución Haitiana que, según Nick Nesbitt, "appears today as the first traumatic announcement of a radically decentered modernity only now (in the increasingly undivided globalization of a new century) becoming fully apparent" (39). Sainte Domingue se manifestó como la expresión de la Ilustración más radical en el world-system de aquel entonces, cuyo impacto sigue siendo percibido hasta hoy día. El impacto global contrasta mucho con los movimientos de la "migración tranquila", como Shalini Puri caracteriza las migraciones intra-caribeñas en la introducción de su volumen sobre Migraciones marginales (2003 1), en el sentido de haber sido tan poco estudiadas en comparación con la diáspora. Sin embargo, al fijarse en el papel del siglo XVIII en las novelas caribeñas escritas en español, se nota un énfasis progresivo en la migración interna. Para Carlos Esteban Deive, por ejemplo, en su novela Viento Negro, Bosque del Caimán (2002), se trata de visualizar las relaciones entre las colonias Sainte Domingue y Santo Domingo en el período de 1790 a 1801, siempre tabuizadas por la historiografía oficial del Trujillato. Deive comienza con el omnipoderoso arzobispo Fernando Portillo y Torres, un personaje histórico, y su indagación de judaizantes en la isla de Curaçao. Pronto se siente rodeado de un clima de judaísmo, masonería, u otras "doctrinas nefastas" procedentes de la Francia Revolucionaria. La novela cubre el período antes y después del Tratado de Basilea en 1795 -cuando los españoles cedieron la parte oriental de Hispaniola a los franceses- terminando con la entrada triunfal de Toussaint Louverture a Santo Domingo, el 26 de enero de 1801. El autor anota en la última frase de su novela que "nunca antes Santo Domingo había estado tan metido en fiesta" (244).

El escritor puertorriqueño Edgardo Rodríguez Juliá también proyecta los acontecimientos en las últimas décadas del siglo XVIII en un marco polémico. Se trata de la transición de la convivencia de "piratas y aventureros ingleses, corsarios franceses, contrabandistas peninsulares y criollos, seis indios de la costa venezolana, cuatro curas españoles amancebados con grifas y tres prostitutas holandesas" (25) hacia una sociedad urbana y criolla, en los años de 1772 a 1797. Los terratenientes aprendieron las lecciones de Adam Smith y de la modernización maquinaria, lo que llevó consigo un incremento acelerado de la necesidad de esclavos africanos. Rodríguez Juliá insiste en que el clima humano y traumático de este período histórico sigue influenciando la contemporaneidad. En una entrevista con Carolina Sancholuz explica:

La primera novela, La renuncia del héroe Baltasar, fue un comienzo juvenil interesante porque no intenté una novela autobiográfica sino que me remonté 
al siglo XVIII, una época distante y oscura de nuestra historia, y fue como un proceso de adivinación de aquello, que Lukács llamaba el "espíritu de una época". Y creo que pude adivinar algunas cosas que luego fueron confirmadas y validadas por la nueva historiografía puertorriqueña. Por un lado, la cuestion del cimarrón -el cimarrón blanco y el cimarrón negro-, la formación de la sociedad puertorriqueña un poco al margen del Estado español. Eso para mi fue muy importante y pienso que en La noche oscura del Niño Avilés también se plantea. La otra adivinación, la cuestión del resentido, esa complejidad que se da en el Caribe, sobre todo en el Caribe mulato, negro, de gente que ha tenido cierto acceso al poder y ha logrado una relación un tanto disparatada respecto de ese poder. (Sancholuz 168)

El momento de transición en La noche oscura del Niño Avilés (1984) se manifiesta por medio del ejemplo del personaje del cronista, el Renegado, quien en contraste con su colega Larra originariamente es un "simpatizante de la causa negra". Como Renegado retrata con precisión las escenas de danzas eróticas, la curación con yerbas o el exorcismo como expresión de montar vuelo hacia "la libertad de su pasado en el gran continente de África" (48). El cambio se realiza cuando el Renegado asume una función oficial como embajador plenipotenciario convirtiéndose en el protagonista de la historia que debe negociar la paz con los cimarrones en su ciudad lacustre. Debido a este cambio de status se rompe cada contacto con la cosmogonía africana de manera brutal, una ruptura que resuena en el estado urbano criollo y cristiano como traumática, como un populismo exorcista y maniático.

En su libro Encuentro con la narrativa dominicana contemporánea (2006) Rita de Maeseneer lleva a cabo una sugestiva lectura contrastiva de la novela de Deive mencionada arriba y del Reino de este Mundo (1949) de Alejo Carpentier. Carpentier sin embargo, ha continuado su investigación narrativa referente a una sociedad urbana construída sobre el trabajo esclavo, centrándose después en La Habana, su ciudad natal. Es posible leer El Reino de este Mundo, El siglo de las luces y La consagración de la primavera como una trilogía, o una serie de novelas por entregas, en la que el problema es la emancipación en combinación con las estructuras internas de una república. También en el siglo XX es difícil manifestarse en el espacio público para los cubanos afrodescendientes, así que Carpentier reclama el derecho de performar sus ceremonias artísticas. Esta reivindicación coincide con la crisis de Playa Girón y la Crisis de los Cohetes cuando Cuba opta por el camino socialista. De este modo, para Carpentier, la Revolución Cubana se entiende en una misma línea como la Revolución Haitiana, como la exigencia de 
una modernidad radical que ahora se enfoca en la transformación de La Habana "poniendo énfasis en su orientación y experiencias personales a nivel internacional, especialmente en los centros europeos" (Phaf 124).

Otra novela sobre el siglo XVIII, Los desertores (De deserteurs 2006), fue escrita por un autor de Curaçao, Frank Martinus Arion. Retrata los tiempos de la independencia norteamericana y las posiciones concernientes a la esclavitud en su discurso público. Como ya dice el título, los protagonistas desertan del ejército patriótico en St. Eustaquio para juntarse a un grupo de cimarrones en la isla vecina, St. Kitts. Uno de ellos, nacido en Barbados e hijo natural de un terrateniente inglés y una mulata de Martinica, delata ante la Corte en St. Kitts lo que había visto en St. Eustaquio. En esta isla holandesa, el gobernador había dado el salut de bienvenida a la marina de los Estados Unidos, así rompiendo el embargo impuesto por los ingleses al comercio con el ejército norteamericano. Esta revelación enfurece tanto al Rey Jorge III en Inglaterra que elabora un plan para destruir Amsterdam, el nervio crucial de la presencia holandesa en América.

Arion tiene una obra novelesca que, al igual que la obra de Carpentier, es posible leer como por entregas. Sin embargo, Los desertores es la única en la que reconstruye acontecimientos en el siglo XVIII. El peligro y la vida en el mar de hombres esclavizados se plantea dentro de una tradición iconográfica y poética que enfatiza las condiciones de transporte en el navío esclavo ${ }^{7}$. Ombre a su vez, plantea su retrospectiva en un marco contemporáneo. En sus reportajes sobre viajes de Holanda a África, el barco de transporte que le lleva a algunas ciudades portuarias da una perspectiva bastante compleja de la vida africana en nuestros días. De acuerdo con Torres-Saillant, se trata aquí de una criollización del centro metropolitano:

Indeed, by carving their niche in the capitals of the intellectural production of the core countries -England, France, Spain, Holland, and the United States- diaspora voices have begun to creolize the imagination of the metropolitan centers, with the foreseeable result that the West may rehabilitate its conventionally inimical portrayal of the Antillean worlds, recognizing its unquestionable humanity and its rightful belonging within the sphere of history. (Torres-Saillant 250)

7 El ícono del "El navío" esclavo data de finales del siglo XVIII (Finley 2004), conoce una variedad de expresiones poéticas en inglés, alemán, francés y el portugués de Brasil en el siglo XIX (Silva 2006); se manifiesta en el arte visual (Finley 2004) así como en la novela contemporánea anglosajona como por ejemplo Mutiny on the Amistad (1987) de Howard Jones y Feeding the Ghosts (1998) de Freddy d'Aguiar. 
Obviamente, la movilidad colonial en la literatura del Caribe holandés efectúa una decentralización criolla al modificar el papel de Amsterdam como capital de la globalización colonizante de antaño. Para esta operación, el desmontaje del siglo XVIII es fundamental porque había diseminado las semillas del drama multicultural momentáneo al ocultar su lógica. Este desmontaje, en el caso de Ellen Ombre, nos lleva al Retour au pays natal, a Paramaribo, donde la autora vive ahora. Sigue publicando sus impresiones contrastivas entre pasado y presente, alternadas de experiencias autobiográficas, con títulos como "Soltado" ( $O p$ drift), "Reciénvenidos" (Nieuwkomers), "Jerusalén bazar" (feruzalem bazaar), y "Música futura" (Toekomstmuziek) (Wie goed 155-188). No será la última etapa de su examinación de alternativas. De todas formas, con Negrojudío en país madre Ombre se sitúa de manera definitiva en la tradición de la négritude, al continuar cuestionando el sentimiento trágico que inhabita su política de neologismos en el siglo XXI.

\section{Obras citadas}

Arion, Frank Martinus. De deserteurs. Amsterdam: De Bezige Bij, 2006.

Berg, Margot van den. A Grammar of Early Sranan. Amsterdam:

$\mathrm{Ph}$ D Dissertation University Amsterdam, 2007.

Böhm, Günther. Los sefardíes en los dominios holandeses de América del Sury del Caribe 1630-1750. Francfort: Vervuert, 1992.

Budick, Emily Miller. Blacks and Jews in Literary Conversation. Cambridge: Cambridge University Press, 1998.

Carpentier, Alejo. El reino de este mundo. México: Siglo XXI, 1949.

-. El siglo de las luces. Barcelona: Barral, 1965.

-. La consagración de la primavera. Barcelona: Barral, 1978.

Clifford, James. "A Politics of Neologism: Aimé Césaire". The Predicament of Culture. Twentieth-Century Ethnography, Litearture, and Art.

Cambridge, Ms.: Harvard University Press, 1988. 175-181.

Crespo Solana, Ana. América desde otra frontera. La Guayana

Holandesa (Surinam): 1680-1795. Madrid: CSIC, 2006.

D’Aguiar, Fred. Feeding the Ghosts. Londres: Cape, 1998.

Deive, Carlos Esteban. Viento Negro, bosque del caimán.

Santo Domingo: Editora Centenario, 2002.

Finley, Cheryl. "Erinnerung verpflichtet. Die Ikone des Sklavenschiffs in der

Vorstellungswelt des Black Atlantic". Der Black Atlantic. Tina Camp y

Paul Gilroy. Ed. Berlin: Haus der Kulturen der Welt, 2004. 248-263. 
Gonçalves, Ana María. Um defeito de cor. Rio de Janeiro/

São Paulo: Editora Record, 2006.

Gyssels, Kathleen. "De slavenroute opnieuw afgelegd: het andere reisverhaal

bij Ellen Ombre en Caryl Phillips". Streven 71.4 (2004): 325-335.

—. "Tristes Tropiques et 'racial healing': Ellen Ombre et Caryl Phillips rentrent au pays". Africa and Its Significant Others: Forty Years of Intercultural Entanglement. Frans-Willem Korsten, Isabel Hoving, y Ernst van Alphen. Eds. Thamyris Intersection 11. Amsterdam / Nueva York: Rodopi, 2003. 163-179.

Israel, Jonathan. The Dutch Republic: Its Rise, Greatness, and

Fall, 1477-1806. Oxford: Clarendon, 1995.

Jones, Howard. Mutiny on the Amistad. Oxford: Oxford University Press, 1987.

MacLeod, Cynthia. Elisabeth Samson. Een vrïe, zwarte vrouw

in het 18e-eeuwse Suriname. Breda: Conserve, 1996.

-. De vrije negerin Elisabeth. Gevangene van kleur. Breda: Conserve, 2000.

Maeseneer, Rita de. "La Hispaniola en la brega con el pasado".

Encuentro con la narrativa dominicana contemporánea. Madrid

/ Francfort: Iberoamericana/Vervuert, 2006. 23-39.

Mirzoeff, Nicholas Ed. Diaspora and Visual Culture. Representing

Africans and fews. Londres: Routledge, 2000.

Nesbitt, Nick. "The Haitian Revolution and the Globalization of the Radical

Enlightenment". Caribbean(s) in the Move-Archipiélagos literarios del Caribe. A

TransArea Symposium. Ottmar Ette Ed. Francfort am Main: Peter Lang, 2008. 39-59.

Ombre, Ellen. "De prijs van hulp". Verhalen van Surinaamse schrijvers.

Michiel van Kempen. Ed. Amsterdam: Arbeiderspers, 1989.

—.Maalstroom. Amsterdam: Arbeiderspers, 1992.

-. Vrouwvreemd. Amsterdam: Arbeiderspers, 1994.

-. Wie goed bedoelt. Amsterdam: Arbeiderspers, 1996.

-. Valse verlangens. Amsterdam: Arbeiderspers, 2000.

-. Negerjood in moederland. Amsterdam: Arbeiderspers, 2004.

-. Wie goed bedoelt: zin + onzin van ontwikkelingshulp.

Amsterdam: Arbeiderspers, 2 ed. Ampliada, 2007.

—. "Juif et nègre au Surinam". Trad. Kathleen Gyssels. Critique 711-712 (2006): 784-785.

—. "Black Jew in the Motherland". Trad. Carolyn Vines. Review.

Literature and Arts of the Americas 40.74 (2007): 101-105.

Palmen, Connie. I. M. (In Memoriam). Amsterdam: Prometheus, 1998.

Phaf, Ineke. "La ciudad y su novela: La Habana". Hacia una historia social de la literatura latinoamericana. Thomas Bremer y Alejandro Losada. Eds. Giessen: Universidad de Giessen, 1985. 
Phaf-Rheinberger, Ineke. "The Contemporary Surinamese Novel". A History of Literature in the Caribbean. A. James Arnold Ed. Vol. 2. Englishand Dutchspeaking Caribbean, subed. Vera Kutzinski \& Ineke Phaf-. Rheinberger. Amsterdam/Filadelfia: John Benjamins, 2001. 527-541.

-. The "Air of Liberty". Narratives of the South Atlantic Past. Amsterdam/ Nueva York: Rodopi, 2008.

Phillips, Caryl. The Transatlantic Sound. Londres: Faber \& Faber, 2000.

Puri, Shalini. "Introduction". Marginal Migrations. The Circulation of Cultures with the Caribbean. Shalini Puri Ed. Warwick: MacMillanPublications, 2003. 1-16.

Rodríguez Juliá, Edgardo. La renuncia del héroe Baltasar. San Juan: Editorial Antillana, 1974 .

-. La noche oscura del Niño Avilés. San Juan: Huracán, 1984.

Sancholuz, Carolina. "Siempre he concebido la literatura muy apegada a la "voz de la tribu'. Entrevista a Edgardo Rodríguez Juliá". Iberoamericana VII. 28 (2008): 168-173.

Scheffer, Paul. "Het multiculturele drama". De multiculturele samenleving.

Disponible en www.nrc.nl/W2/Lab/Multicultureel/scheffer.html

Schorsch, Jonathan. Fews and Blacks in the Early Modern World.

Cambridge: Cambridge University Press, 2004.

Silva, Alberto da Costa. Castro Alves: um poeta sempre jovem.

São Paulo: Companhia das Letras, 2006.

Torres-Saillant, Silvio. An Intellectual History of the Caribbean.

Nueva York: Palgrave Macmillan, 2006. 